Article

\title{
Informal Complete Metric Space and Fixed Point Theorems
}

\author{
Hsien-Chung Wu \\ Department of Mathematics, National Kaohsiung Normal University, Kaohsiung 802, Taiwan; \\ hcwu@nknucc.nknu.edu.tw
}

Received: 19 October 2019; Accepted: 2 November 2019; Published: 7 November 2019

\begin{abstract}
The concept of informal vector space is introduced in this paper. In informal vector space, the additive inverse element does not necessarily exist. The reason is that an element in informal vector space which subtracts itself cannot be a zero element. An informal vector space can also be endowed with a metric to define a so-called informal metric space. The completeness of informal metric space can be defined according to the similar concept of a Cauchy sequence. A new concept of fixed point and the related results are studied in informal complete metric space.
\end{abstract}

Keywords: Cauchy sequence; near fixed point; informal metric space; informal vector space; null set

MSC: 47H10; 54H25

\section{Introduction}

The basic operations in (conventional) vector space are vector addition and scalar multiplication. Based on these two operations, the vector space should satisfy some required conditions (eight axioms in total) by referring to [1-5]. However, some spaces cannot comply with all of the axioms given in vector space. For example, the space consisting of all subsets of $\mathbb{R}$ cannot satisfy all of the axioms in vector space (Wu [6]). Also, the space consisting of all fuzzy numbers in $\mathbb{R}$ cannot satisfy all of the axioms in vector space, where the addition and scalar multiplication of fuzzy sets are considered $(\mathrm{Wu}$ [7]). The main reason is that the additive inverse element does not exist.

Let $S$ and $T$ be two subsets of $\mathbb{R}$. The addition and scalar multiplication for the subsets of $\mathbb{R}$ are defined by

$$
S+T=\{s+t: s \in S \text { and } t \in T\} \text { and } k S=\{k s: s \in S\} \text { for any } k \in \mathbb{R} \text {. }
$$

Let $\mathcal{X}$ denote the family of all subsets of $\mathbb{R}$. Given any $S \in \mathcal{X}$, the subtraction $S-S$ by itself is given by

$$
S-S=\left\{s_{1}-s_{2}: s_{1}, s_{2} \in S\right\},
$$

which cannot be the zero element in $\mathcal{X}$. Therefore, in this paper, we propose the concept of null set for the purpose of playing the role of a zero element in the so-called informal vector space. Since the informal metric space is a completely new concept, there are no available, relevant references for this topic. The readers may instead refer to the monographs [1-5] on topological vector spaces and the monographs [8-10] on functional analysis.

In this paper, we propose the concept of informal vector space that can include the space consisting of all bounded and closed intervals in $\mathbb{R}$ and the space consisting of all fuzzy numbers in $\mathbb{R}$. We also introduce the concept of null set that can be regarded as a kind of "zero element" of informal vector space. When the null set is degenerated as a singleton set $\{\theta\}$, an informal vector space will turn into a conventional vector space with the zero element $\theta$. In other words, the results obtained in 
this paper can be reduced to the results in conventional vector space when the null set is taken to be a singleton set.

Based on the concept of null set, we can define the concept of almost identical elements in informal vector space. We can also endow a metric to the informal vector space defining the so-called informal metric space. This kind of metric is completely different from the conventional metric defined in vector space, since it involves the null set and almost identical concept. The most important triangle inequality is still included in an informal metric space. Based on this metric, the concepts of limit and class limit of a sequence in informal metric space are defined herein. Under this setting, we can similarly define the concept of a Cauchy sequence, which can be used to define the completeness of informal metric space. The main aim of this paper was to establish the so-called near-fixed point in informal, complete metric space, where the near fixed point is based on the almost identical concept. We shall also claim that if the null set is degenerated as a singleton set, then the concept of near a fixed point is identical to the concept of a (conventional) fixed point.

In Sections 2 and 3, the concept of informal vector space and informal metric space are proposed. The interesting properties are derived in order to study the new type of fixed point theorems. In Section 4, according to the informal metric, the concept of a Cauchy sequence is similarly defined. The completeness of informal metric space is also defined according to the concept of Cauchy sequences. In Section 5, we present many new types of fixed point theorems that are established using the almost identical concept in informal metric space.

\section{Informal Vector Spaces}

Let $X$ be a universal set, and let $\mathbb{F}$ be a scalar field. We assume that $X$ is endowed with the vector addition $x \oplus y$ and scalar multiplication $\alpha x$ for any $x, y \in X$ and $\alpha \in \mathbb{F}$. In this case, we call $X$ a universal set over $\mathbb{F}$. In the conventional vector space over $\mathbb{F}$, the additive inverse element of $x$ is denoted by $-x$, and it can also be shown that $-x=-1 x$. In this paper, we shall not consider the concept of inverse elements. However, for convenience, we still adopt $-x=-1 x$.

For $x, y \in X$, the substraction $x \ominus y$ is defined by $x \ominus y=x \oplus(-y)$, where $-y$ means the scalar multiplication $(-1) y$. For any $x \in X$ and $\alpha \in \mathbb{F}$, we have to mention that $(-\alpha) x \neq-\alpha x$ and $\alpha(-x) \neq-\alpha x$ in general, unless $\alpha(\beta x)=(\alpha \beta) x$ for any $\alpha, \beta \in \mathbb{F}$. In this paper, this law will not always be assumed to be true.

Example 1. Let $C$ be a subset of complex plane $\mathbb{C}$ defined by

$$
C=\{a+b i: a, b \in \mathbb{R} \text { satisfying } a \leq b\} .
$$

The usual addition and scalar multiplication in $\mathbb{C}$ are defined by

$$
(a+b i)+(c+d i)=(a+c)+(b+d) i \text { and } k(a+b i)=k a+k b i \text { for } k \in \mathbb{R} .
$$

Given any $z=a+b i \in C$, its additive inverse in $\mathbb{C}$ denoted by $-z$ is

$$
-z=(-1) z=-a-b i .
$$

We see that $-z \notin C$. Therefore, the subset $C$ is not closed under the above scalar multiplication. In other words, the subset $C$ cannot form a vector space. However, if the scalar multiplication in the subset $C$ is defined by

$$
k(a+b i)= \begin{cases}k a+k b i & \text { if } k \geq 0 \\ k b+k a i & \text { if } k<0\end{cases}
$$

then the subset $C$ is closed under the above addition and this new scalar multiplication. In this case, we shall consider the subset $C$ as an informal vector space that will be defined below. 
Example 2. Let $\mathcal{I}$ be the set of all closed intervals in $\mathbb{R}$. The addition is given by

$$
[a, b] \oplus[c, d]=[a+c, b+d]
$$

and the scalar multiplication is given by

$$
k[a, b]= \begin{cases}{[k a, k b]} & \text { if } k \geq 0 \\ {[k b, k a]} & \text { if } k<0 .\end{cases}
$$

We see that $\mathcal{I}$ cannot be a (conventional) vector space, since the inverse element cannot exist for any non-degenerated closed interval. On the other hand, the distributive law for scalar addition does not hold true in $\mathcal{I}$; that is, the equality $(\alpha+\beta) x=\alpha x \oplus \beta x$ cannot hold true for any $x \in \mathcal{I}$ and $\alpha, \beta \in \mathbb{R}$. This shows another reason why $\mathcal{I}$ cannot be a (conventional) vector space.

Definition 1. Let $X$ be a universal set over the scalar field $\mathbb{F}$. We define the null set of $X$ as follows

$$
\Omega=\{x \ominus x: x \in X\} .
$$

We say that the null set $\Omega$ satisfies the neutral condition if and only if $\omega \in \Omega$ implies $-\omega \in \Omega$.

Example 3. Continued from Example 1, for any $z=a+b i \in C$, we have

$$
z \ominus z=z+(-1) z=(a+b i)+(-b-a i)=(a-b)+(b-a) i \in C .
$$

Therefore, the null set $\Omega$ is given by

$$
\Omega=\{-k+k i: k \in \mathbb{R} \text { and } k \geq 0\}=\left\{-k+k i: k \in \mathbb{R}_{+}\right\}
$$

Now we are in a position to define the concept of informal vector space.

Definition 2. Let $X$ be a universal set over $\mathbb{F}$. We say that $X$ is an informal vector space over $\mathbb{F}$ if and only if the following conditions are satisfied:

- $1 x=x$ for any $x \in X$;

- $\quad x=y$ implies $x \oplus z=y \oplus z$ and $\alpha x=\alpha y$ for any $x, y, z \in X$ and $\alpha \in \mathbb{F}$;

- The commutative and associative laws for vector addition hold true in $X$; that is, $x \oplus y=y \oplus x$ and $(x \oplus y) \oplus z=x \oplus(y \oplus z)$ for any $x, y, z \in X$.

Definition 3. Let $X$ be an informal vector space over $\mathbb{F}$ with the null set $\Omega$. Given any $x, y \in X$, we say that $x$ and $y$ are almost identical if and only if any one of the following conditions is satisfied:

- $x=y$;

- $\quad$ There exists $\omega \in \Omega$ such that $x=y \oplus \omega$ or $x \oplus \omega=y$;

- $\quad$ There exists $\omega_{1}, \omega_{2} \in \Omega$ such that $x \oplus \omega_{1}=y \oplus \omega_{2}$.

In this case, we write $x \stackrel{\Omega}{=} y$.

Remark 1. Suppose that the informal vector space $X$ over $\mathbb{F}$ with the null set $\Omega$ contains the zero element $\theta$; that is, $x=x \oplus \theta=\theta \oplus x$ for any $x \in X$. Then, we can simply say that $x \stackrel{\Omega}{=} y$ if and only if $\omega_{1}, \omega_{2} \in \Omega$ exists, such that $x \oplus \omega_{1}=y \oplus \omega_{2}$ (i.e., only the third condition is satisfied), since the first and second conditions can be rewritten as the third condition by adding the zero element $\theta$. We also remark that if we want to discuss some properties based on $x \stackrel{\Omega}{=} y$, it suffices to consider the third condition $x \oplus \omega_{1}=y \oplus \omega_{2}$, even though $X$ does not contain the zero element $\theta$. The reason is that the same arguments are still applicable for the first and second conditions. 
According to the binary relation $\stackrel{\Omega}{=}$, for any $x \in X$, we define the class

$$
[x]=\{y \in X: x \stackrel{\Omega}{=} y\} .
$$

The family of all classes $[x]$ for $x \in X$ is denoted by $[X]$. For $y \in[x]$, it is not necessarily that the class $[y]$ is equal to the class $[x]$, unless the binary relation $\stackrel{\Omega}{=}$ is an equivalence relation.

Proposition 1. Let $X$ be an informal vector space over $\mathbb{F}$ with the null set $\Omega$. If $\Omega$ is closed under the vector addition, then the binary relation $\Omega$ is an equivalence relation.

Proof. For any $x \in X, x=x$ implies $x \stackrel{\Omega}{=} x$, which shows the reflexivity. According to the definition of the binary relation $\stackrel{\Omega}{=}$, the symmetry is obvious. Regarding the transitivity, for $x \stackrel{\Omega}{=} y$ and $y \stackrel{\Omega}{=} z$, we want to claim $x \stackrel{\Omega}{=} z$. From Remark 1, it suffices to just consider the cases of

$$
x \oplus \omega_{1}=y \oplus \omega_{2} \text { and } y \oplus \omega_{3}=z \oplus \omega_{4}
$$

for some $\omega_{i} \in \Omega$ for $i=1, \cdots, 4$. By the associative and commutative laws for vector addition, we have

$$
x \oplus \omega_{1} \oplus \omega_{3}=y \oplus \omega_{3} \oplus \omega_{2}=z \oplus \omega_{4} \oplus \omega_{2}
$$

which shows $x \stackrel{\Omega}{=} z$, since $\Omega$ is closed under the vector addition. This completes the proof.

Let $X$ be an informal vector space over $\mathbb{F}$ with the null set $\Omega$ such that $\Omega$ is closed under the vector addition. Proposition 1 says that the classes defined in (1) form the equivalence classes. It is clear to see that $y \in[x]$ implies $[x]=[y]$. In other words, the family of all equivalence classes form a partition of the whole set $X$.

We also need to remark that the space $[X]$ is still not a (conventional) vector space. The reason is that not all of the axioms taken in the vector space will be satisfied in $[X]$, since the original space $X$ does not satisfy all of the axioms in the vector space. For example, we consider the informal vector space $\mathcal{I}$ over $\mathbb{R}$ from Example 2. The quotient set $[\mathcal{I}]$ cannot be a real vector space, since

$$
(\alpha+\beta)[x] \neq \alpha[x]+\beta[x]
$$

for $\alpha \beta<0$. The reason is that $(\alpha+\beta) x \neq \alpha x+\beta x$ for $x \in \mathcal{I}$ and $\alpha \beta<0$.

\section{Informal Metric Spaces}

Now, we are in a position to introduce the concept of the so-called informal metric space.

Definition 4. Let $X$ be an informal vector space over $\mathbb{F}$ with the null set $\Omega$. For the non-negative, real-valued function d defined on $X \times X$, we consider the following conditions:

(i) $\quad d(x, y)=0$ if and only if $x \stackrel{\Omega}{=} y$ for all $x, y \in X$;

(i') $d(x, y)=0$ if and only if $x=y$ for all $x, y \in X$;

(ii) $d(x, y)=d(y, x)$ for all $x, y \in X$;

(iii) $d(x, y) \leq d(x, z)+d(z, y)$ for all $x, y, z \in X$.

Different kinds of metric spaces are defined below.

- The pair $(X, d)$ is called a pseudo-metric space if and only if d satisfies conditions (ii) and (iii).

- The pair $(X, d)$ is called a metric space if and only if $d$ satisfies conditions ( $\left.i^{\prime}\right)$, (ii), and (iii).

- The pair $(X, d)$ is called a informal metric space if and only if d satisfies conditions (i), (ii), and (iii). 
(iv) We say that $d$ satisfies the null super-inequality if and only if, for any $\omega_{1}, \omega_{2} \in \Omega$ and $x, y, z \in X$, we have

$$
d\left(x \oplus \omega_{1}, y \oplus \omega_{2}\right) \geq d(x, y), \quad d\left(x \oplus \omega_{1}, y\right) \geq d(x, y) \text { and } d\left(x, y \oplus \omega_{2}\right) \geq d(x, y)
$$

(iv') We say that $d$ satisfies the null sub-inequality if and only if, for any $\omega_{1}, \omega_{2} \in \Omega$ and $x, y \in X$, we have

$$
d\left(x \oplus \omega_{1}, y \oplus \omega_{2}\right) \leq d(x, y), \quad d\left(x \oplus \omega_{1}, y\right) \leq d(x, y) \text { and } d\left(x, y \oplus \omega_{2}\right) \leq d(x, y) .
$$

(iv") We say that $d$ satisfies the null equality if and only if, for any $\omega_{1}, \omega_{2} \in \Omega$ and $x, y \in X$, we have

$$
d\left(x \oplus \omega_{1}, y \oplus \omega_{2}\right)=d(x, y), \quad d\left(x \oplus \omega_{1}, y\right)=d(x, y) \quad \text { and } \quad d\left(x, y \oplus \omega_{2}\right)=d(x, y)
$$

Example 4. Continued from Example 2, we take $X=\mathcal{I}$ that consists of all bounded and closed intervals, which is not a vector space. For $I_{1}=\left[a_{1}^{L}, a_{1}^{U}\right]$ and $I_{2}=\left[a_{2}^{L}, a_{2}^{U}\right]$ in $\mathcal{I}$, we define a nonnegative real-valued function $d$ for $\mathcal{I} \times \mathcal{I}$ by

$$
d\left(I_{1}, I_{2}\right)=\left|a_{1}^{L}+a_{1}^{U}-a_{2}^{L}-a_{2}^{U}\right|
$$

Suppose that

$$
d\left(I_{1}, I_{2}\right)=\left|a_{1}^{L}+a_{1}^{U}-a_{2}^{L}-a_{2}^{U}\right|=0 .
$$

We cannot obtain $I_{1}=I_{2}$. Therefore, condition $\left(i^{\prime}\right)$ in Definition 4 is not satisfied, which says that $(\mathcal{I}, d)$ cannot be a (standard) metric space. However, using the basic arithmetics, we can obtain $I_{1} \stackrel{\Omega}{=} I_{2}$. For any $I_{1}, I_{2}, I_{3}$ in $\mathcal{I}$, it is not difficult to show that

$$
d\left(I_{1}, I_{2}\right)=d\left(I_{2}, I_{1}\right) \text { and } d\left(I_{1}, I_{2}\right) \leq d\left(I_{1}, I_{3}\right)+d\left(I_{3}, I_{2}\right)
$$

Therefore $(\mathcal{I}, d)$ is indeed an informal metric space. Moreover, we are going to claim that $d$ also satisfies the null equality. We first note that the null set $\Omega$ in $\mathcal{I}$ is given by

$$
\Omega=\{[-k, k]: k \geq 0\}
$$

For any $k_{1}, k_{2} \in \mathbb{R}_{+}$, i.e., $\omega_{1}=\left[-k_{1}, k_{1}\right], \omega_{2}=\left[-k_{2}, k_{2}\right] \in \Omega$, we have

$$
\begin{aligned}
d\left(I_{1} \oplus \omega_{1}, I_{2} \oplus \omega_{2}\right)= & d\left(\left[a_{1}^{L}, a_{1}^{U}\right] \oplus\left[-k_{1}, k_{1}\right],\left[a_{2}^{L}, a_{2}^{U}\right] \oplus\left[-k_{2}, k_{2}\right]\right) \\
& =d\left(\left[a_{1}^{L}-k_{1}, a_{1}^{U}+k_{1}\right],\left[a_{2}^{L}-k_{2}, a_{2}^{U}+k_{2}\right]\right) \\
& =\left|\left(a_{1}^{L}-k_{1}+a_{1}^{U}+k_{1}\right)-\left(a_{2}^{L}-k_{2}+a_{2}^{U}+k_{2}\right)\right| \\
& =\left|\left(a_{1}^{L}+a_{1}^{U}\right)-\left(a_{2}^{L}+a_{2}^{U}\right)\right| \\
& =d\left(\left[a_{1}^{L}, a_{1}^{U}\right],\left[a_{2}^{L}, a_{2}^{U}\right]\right)=d\left(I_{1}, I_{2}\right),
\end{aligned}
$$

which shows that $d$ indeed satisfies the null equality.

\section{Cauchy Sequences}

In this section, we are going to introduce the concepts of Cauchy sequences and completeness in the informal metric space. We first introduce the concept of limit in the informal metric space.

Definition 5. Let $X$ be an informal vector space over $\mathbb{F}$ with the null set $\Omega$, and let $(X, d)$ be a pseudo-metric space. The sequence $\left\{x_{n}\right\}_{n=1}^{\infty}$ in $X$ is said to be convergent if and only if

$$
\lim _{n \rightarrow \infty} d\left(x_{n}, x\right)=0 \text { for some } x \in X \text {. }
$$


The element $x$ is called the limit of the sequence $\left\{x_{n}\right\}_{n=1}^{\infty}$.

The sense of uniqueness of limit will be different for the metric space and informal metric space. Let $\left\{x_{n}\right\}_{n=1}^{\infty}$ be a sequence in $(X, d)$. If there exists $x, y \in X$ such that

$$
\lim _{n \rightarrow \infty} d\left(x_{n}, x\right)=0=\lim _{n \rightarrow \infty} d\left(x_{n}, y\right)
$$

then, by the triangle inequality (iii) in Definition 4 , we have

$$
0 \leq d(x, y) \leq d\left(x, x_{n}\right)+d\left(x_{n}, y\right) \rightarrow 0+0=0 \text { as } n \rightarrow \infty,
$$

which says that $d(x, y)=0$.

- Suppose that $(X, d)$ is a metric space. By condition $\left(\mathrm{i}^{\prime}\right)$ in Definition 4 , we see that $x=y$. This shows the uniqueness.

- Suppose that $(X, d)$ is an informal metric space. By condition (i) in Definition 4 , we see that $x \stackrel{\Omega}{=} y$. Recall that if $\Omega$ is closed under the vector addition, then we can consider the equivalence classes. In this case, we also see that $y$ is in the equivalence class $[x]$.

On the other hand, we further assume that $d$ satisfies the null equality. If $\left\{x_{n}\right\}_{n=1}^{\infty}$ is a sequence in $X$ such that $d\left(x_{n}, x\right) \rightarrow 0$ as $n \rightarrow \infty$, then, for any $y \in[x]$, i.e., $x \oplus \omega_{1}=y \oplus \omega_{2}$ for some $\omega_{1}, \omega_{2} \in \Omega$, we also have $d\left(x_{n}, y\right) \rightarrow 0$ as $n \rightarrow \infty$, as shown below:

$$
0 \leq d\left(x_{n}, y\right)=d\left(x_{n}, \omega_{2} \oplus y\right)=d\left(x_{n}, \omega_{1} \oplus x\right)=d\left(x_{n}, x\right) \rightarrow 0 \text { as } n \rightarrow \infty
$$

Therefore, we propose the following definition.

Definition 6. Let $(X, d)$ be an informal pseudo-metric space with the null set $\Omega$. If $\left\{x_{n}\right\}_{n=1}^{\infty}$ is a sequence in X such that

$$
\lim _{n \rightarrow \infty} d\left(x_{n}, x\right)=0
$$

for some $x \in X$, then the class $[x]$ is called the class limit of $\left\{x_{n}\right\}_{n=1}^{\infty}$. We also write

$$
\lim _{n \rightarrow \infty} x_{n}=[x] \text { or } x_{n} \rightarrow[x]
$$

Proposition 2. Let $(X, d)$ be an informal pseudo-metric space with the null set $\Omega$ such that $\Omega$ is closed under the vector addition. Then, the class limit in the informal metric space is unique.

Proof. Let $\left\{x_{n}\right\}_{n=1}^{\infty}$ be a convergent sequence in $X$ with the class limits $[x]$ and $[y]$. According to the definition, we have

$$
\lim _{n \rightarrow \infty} d\left(x_{n}, x\right)=0 \text { and } \lim _{n \rightarrow \infty} d\left(x_{n}, y\right)=0 .
$$

Using (2), it follows that $d(x, y)=0$, which also implies $y \in[x]$, i.e., $[x]=[y]$. This shows the uniqueness in the sense of class limit.

Definition 7. Let $(X, d)$ be an informal metric space.

- A sequence $\left\{x_{n}\right\}_{n=1}^{\infty}$ in $X$ is called a Cauchy sequence if and only if, given any $\epsilon>0, N \in \mathbb{N}$ exists, such that $d\left(x_{n}, x_{m}\right)<\epsilon$ for all $n>N$ and $m>N$.

- A subset $M$ of $X$ is said to be complete if and only if every Cauchy sequence in $M$ is convergent to some element in $M$.

Proposition 3. Every convergent sequence in an informal metric space is a Cauchy sequence. 
Example 5. Continued from Example 4, we see that $(\mathcal{I}, d)$ is an informal metric space such that $d$ satisfies the null equality. We are going to claim that $(\mathcal{I}, d)$ is complete. Given a sequence $\left\{I_{n}\right\}_{n=1}^{\infty}$ in the informal metric space $(\mathcal{I}, d)$ by $I_{n}=\left[a_{n}^{L}, a_{n}^{U}\right]$ for $n=1,2, \cdots$, suppose that $\left\{I_{n}\right\}_{n=1}^{\infty}$ is a Cauchy sequence. Then, given any $\epsilon>0$, for sufficiently large $n$ and $m$, we have

$$
\epsilon>d\left(I_{n}, I_{m}\right)=d\left(\left[a_{n}^{L}, a_{n}^{U}\right],\left[a_{m}^{L}, a_{m}^{U}\right]\right)=\left|\left(a_{n}^{L}+a_{n}^{U}\right)-\left(a_{m}^{L}+a_{m}^{U}\right)\right|
$$

Let $a_{n}=a_{n}^{L}+a_{n}^{U}$. Then, the expression (3) shows that $\left\{a_{n}\right\}_{n=1}^{\infty}$ is a Cauchy sequence in $\mathbb{R}$. The completeness of $\mathbb{R}$ says that $a \in \mathbb{R}$ exists, satisfying $\left|a_{n}-a\right|<\epsilon$ for sufficiently large $n$. Now we define a bounded closed interval $\left[a^{L}, a^{U}\right]$ satisfying $a^{L}+a^{U}=a$. Then

$$
d\left(\left[a_{n}^{L}, a_{n}^{U}\right],\left[a^{L}, a^{U}\right]\right)=\left|\left(a_{n}^{L}+a_{n}^{U}\right)-\left(a^{L}+a^{U}\right)\right|=\left|a_{n}-a\right|<\epsilon
$$

for a sufficiently large $n$, which says that the sequence $\left\{I_{n}\right\}_{n=1}^{\infty}$ converges to $\left[a^{L}, a^{U}\right]$. Therefore, we conclude that the space $(\mathcal{I}, d)$ is complete.

\section{Near Fixed Point Theorems}

Let $X$ be a universal set, and let $T: X \rightarrow X$ be a function from $X$ into itself. We say that $x \in X$ is a fixed point if and only if $T(x)=x$. The well-known Banach contraction principle presents the fixed point of function $T$ when $X$ is taken to be a complete metric space. We shall study the Banach contraction principle when $X$ is taken to be an informal complete metric space.

Definition 8. Let $X$ be an informal vector space over $\mathbb{F}$ with a null set $\Omega$, and let $T: X \rightarrow X$ be a function defined on $X$ into itself. A point $x \in X$ is called a near fixed point of $T$ if and only if $T(x) \stackrel{\Omega}{=} x$.

Example 6. Continued from Example 5, we see that the null set $\Omega$ in $(\mathcal{I}, d)$ is given by

$$
\Omega=\{[-k, k]: k \geq 0\}
$$

which is closed under the vector addition. Let $T:(\mathcal{I}, d) \rightarrow(\mathcal{I}, d)$ be a function from $\mathcal{I}$ into itself. Suppose that $\left[a^{L}, a^{U}\right]$ is a near fixed point of $T$, i.e., $T\left(\left[a^{L}, a^{U}\right]\right) \stackrel{\Omega}{=}\left[a^{L}, a^{U}\right]$. By definition, nonnegative numbers $k_{1}$ and $k_{2}$ exist such that one of the following equalities is satisfied:

- $\quad T\left(\left[a^{L}, a^{U}\right]\right) \oplus\left[-k_{1}, k_{1}\right]=\left[a^{L}, a^{U}\right] ;$

- $T\left(\left[a^{L}, a^{U}\right]\right)=\left[a^{L}, a^{U}\right] \oplus\left[-k_{1}, k_{1}\right]$

- $\quad T\left(\left[a^{L}, a^{U}\right]\right) \oplus\left[-k_{1}, k_{1}\right]=\left[a^{L}, a^{U}\right] \oplus\left[-k_{2}, k_{2}\right]$,

where $\left[-k_{1}, k_{1}\right]$ and $\left[-k_{2}, k_{2}\right]$ are in the null set $\Omega$.

Remark 2. We have the following observations.

- By definition, we see that $T(x) \stackrel{\Omega}{=} x$ if and only if $\omega_{1}, \omega_{2} \in \Omega$ exist, such that $T(x)=x, T(x) \oplus \omega_{1}=x$, or $T(x)=x \oplus \omega_{1}$ or $T(x) \oplus \omega_{1}=x \oplus \omega_{2}$.

- If the informal vector space X owns a zero element $\theta$, then the (conventional) fixed point is also a near fixed point.

- If the informal vector space X turns into a (conventional) vector space over $\mathbb{F}$, then the concepts of near fixed point and (conventional) fixed point are equivalent.

Definition 9. Let $(X, d)$ be an informal metric space. A function $T:(X, d) \rightarrow(X, d)$ is called a contraction of $X$ if and only if there is a real number $0<\alpha<1$ such that

$$
d(T(x), T(y)) \leq \alpha d(x, y)
$$


for any $x, y \in X$.

Example 7. Continued from Example 4, suppose that $T$ is a contraction of $\mathcal{I}$. Then, a real number $0<\alpha<1$ exists, such that

$$
\left.d\left(T\left(\left[a_{1}^{L}, a_{1}^{U}\right]\right), T\left(a_{2}^{L}, a_{2}^{U}\right]\right)\right) \leq \alpha \cdot d\left(\left[a_{1}^{L}, a_{1}^{U}\right],\left[a_{2}^{L}, a_{2}^{U}\right]\right)
$$

for any $\left[a_{1}^{L}, a_{1}^{U}\right],\left[a_{2}^{L}, a_{2}^{U}\right] \in \mathcal{I}$. In particular, we take $\overline{\mathcal{I}}$ to be a collection of all subintervals of $[0,1]$. Now, we take $T: \overline{\mathcal{I}} \rightarrow \overline{\mathcal{I}}$ by

$$
\begin{aligned}
T\left(\left[a^{L}, a^{U}\right]\right) & =\frac{1}{3}\left[a^{L}, a^{U}\right] \otimes\left[a^{L}, a^{U}\right] \\
& =\frac{1}{3}\left[\min \left\{a^{L} a^{L}, a^{L} a^{U}, a^{U} a^{U}\right\}, \max \left\{a^{L} a^{L}, a^{L} a^{U}, a^{U} a^{U}\right\}\right]=\frac{1}{3}\left[a^{L} a^{L}, a^{U} a^{U}\right],
\end{aligned}
$$

where $a^{L}, a^{U} \in[0,1]$. From Example 4, we have

$$
\begin{aligned}
\left.d\left(T\left(\left[a_{1}^{L}, a_{1}^{U}\right]\right), T\left(a_{2}^{L}, a_{2}^{U}\right]\right)\right) & =d\left(\frac{1}{3}\left[a_{1}^{L} a_{1}^{L}, a_{1}^{U} a_{1}^{U}\right], \frac{1}{3}\left[a_{2}^{L} a_{2}^{L}, a_{2}^{U} a_{2}^{U}\right]\right) \\
& =\frac{1}{3}\left|a_{1}^{L} a_{1}^{L}+a_{1}^{U} a_{1}^{U}-a_{2}^{L} a_{2}^{L}-a_{2}^{U} a_{2}^{U}\right| \\
& =\frac{1}{3}\left|\left(a_{1}^{L}+a_{2}^{L}\right)\left(a_{1}^{L}-a_{2}^{L}\right)+\left(a_{1}^{U}+a_{2}^{U}\right)\left(a_{1}^{U}-a_{2}^{U}\right)\right| \\
& \leq \frac{2}{3}\left|\left(a_{1}^{L}-a_{2}^{L}\right)+\left(a_{1}^{U}-a_{2}^{U}\right)\right|\left(\text { since } a_{1}^{L}, a_{1}^{U}, a_{2}^{L}, a_{2}^{U} \in[0,1]\right) \\
& =\frac{2}{3} d\left(\left[a_{1}^{L}, a_{1}^{U}\right],\left[a_{2}^{L}, a_{2}^{U}\right]\right),
\end{aligned}
$$

which says that $T$ is a contraction of $\overline{\mathcal{I}}$.

Given any initial element $x_{0} \in X$, we define the iterative sequence $\left\{x_{n}\right\}_{n=1}^{\infty}$ using the function $T$ as follows:

$$
x_{1}=T\left(x_{0}\right), \quad x_{2}=T\left(x_{1}\right)=T^{2}\left(x_{0}\right), \cdots, x_{n}=T^{n}\left(x_{0}\right) .
$$

Under some suitable conditions, we are going to show that the sequence $\left\{x_{n}\right\}_{n=1}^{\infty}$ can converge to a near fixed point.

Theorem 1. Let $(X, d)$ be an informal complete metric space with the null set $\Omega$ such that $d$ satisfies the null equality. Suppose that $\Omega$ is closed under the vector addition, and that the function $T:(X, d) \rightarrow(X, d)$ is a contraction of $X$. Then $T$ has a near fixed point $x \in X$ satisfying $T(x) \stackrel{\Omega}{=} x$. More precisely, the near fixed point $x$ can be obtained by the following limit

$$
d\left(x_{n}, x\right) \rightarrow 0 \text { as } n \rightarrow \infty,
$$

where the sequence $\left\{x_{n}\right\}_{n=1}^{\infty}$ is generated by the iteration (4). Moreover, we have the following properties.

- There is a unique equivalence class $[x]$ satisfying that if $\bar{x} \notin[x]$, then $\bar{x}$ cannot be a near fixed point, which shows the sense of uniqueness.

- Suppose that $\bar{x} \in[x]$. Then $\bar{x}$ is also a near fixed point of $T$ satisfying $T(\bar{x}) \stackrel{\Omega}{=} \bar{x}$ and $[\bar{x}]=[x]$.

- Suppose that $\bar{x}$ is a near fixed point of T. Then $\bar{x} \in[x]$; i.e., $[\bar{x}]=[x]$. In other words, if $x$ and $\bar{x}$ are the near fixed points of $T$, then $x \stackrel{\Omega}{=} \bar{x}$. 
Proof. Proposition 1 says that the family of all classes $[x]$ for $x \in X$ forms the equivalence classes. Given any initial element $x_{0} \in X$, we can generate the iterative sequence $\left\{x_{n}\right\}_{n=1}^{\infty}$ using (4). We want to claim that $\left\{x_{n}\right\}_{n=1}^{\infty}$ is a Cauchy sequence. Since $T$ is a contraction of $X$, we have

$$
\begin{aligned}
d\left(x_{m+1}, x_{m}\right) & =d\left(T\left(x_{m}\right), T\left(x_{m-1}\right)\right) \leq \alpha d\left(x_{m}, x_{m-1}\right) \\
& =\alpha d\left(T\left(x_{m-1}\right), T\left(x_{m-2}\right)\right) \leq \alpha^{2} d\left(x_{m-1}, x_{m-2}\right) \\
& \leq \cdots \leq \alpha^{m} d\left(x_{1}, x_{0}\right) .
\end{aligned}
$$

For $n<m$, using the triangle inequality, we obtain

$$
\begin{aligned}
d\left(x_{m}, x_{n}\right) & \leq d\left(x_{m}, x_{m-1}\right)+d\left(x_{m-1}, x_{m-2}\right)+\cdots+d\left(x_{n+1}, x_{n}\right) \\
& \leq\left(\alpha^{m-1}+\alpha^{m-2}+\cdots+\alpha^{n}\right) \cdot d\left(x_{1}, x_{0}\right) \\
& =\alpha^{n} \cdot \frac{1-\alpha^{m-n}}{1-\alpha} \cdot d\left(x_{1}, x_{0}\right) .
\end{aligned}
$$

Since $0<\alpha<1$, we have $1-\alpha^{m-n}<1$ in the numerator, which says that

$$
d\left(x_{m}, x_{n}\right) \leq \frac{\alpha^{n}}{1-\alpha} \cdot d\left(x_{1}, x_{0}\right) \rightarrow 0 \text { as } n \rightarrow \infty .
$$

This shows that $\left\{x_{n}\right\}_{n=1}^{\infty}$ is indeed a Cauchy sequence. The completeness says that $x \in X$ exists, satisfying $d\left(x_{n}, x\right) \rightarrow 0$, i.e., $x_{n} \rightarrow[x]$ from Definition 6 and Proposition 2 .

Now, we want to claim that any point $\bar{x} \in[x]$ is a near fixed point. We first have $\bar{x} \oplus \omega_{1}=x \oplus \omega_{2}$ for some $\omega_{1}, \omega_{2} \in \Omega$. According to the triangle inequality and using the fact of contraction of $X$, we obtain

$$
\begin{aligned}
d(\bar{x}, T(\bar{x})) & =d\left(\bar{x} \oplus \omega_{1}, T(\bar{x})\right) \text { (since } d \text { satisfies the null equality) } \\
& \leq d\left(\bar{x} \oplus \omega_{1}, x_{m}\right)+d\left(x_{m}, T(\bar{x})\right) \\
& =d\left(\bar{x} \oplus \omega_{1}, x_{m}\right)+d\left(T\left(x_{m-1}\right), T(\bar{x})\right) \\
& \leq d\left(\bar{x} \oplus \omega_{1}, x_{m}\right)+\alpha d\left(x_{m-1}, \bar{x}\right) \\
& =d\left(\bar{x} \oplus \omega_{1}, x_{m}\right)+\alpha d\left(x_{m-1}, \bar{x} \oplus \omega_{1}\right) \text { (since } d \text { satisfies the null equality) } \\
& =d\left(x \oplus \omega_{2}, x_{m}\right)+\alpha d\left(x_{m-1}, x \oplus \omega_{2}\right) \\
& =d\left(x, x_{m}\right)+\alpha d\left(x_{m-1}, x\right) \text { (since } d \text { satisfies the null equality), }
\end{aligned}
$$

which implies $d(\bar{x}, T(\bar{x}))=0$ as $m \rightarrow \infty$. We conclude that $T(\bar{x}) \stackrel{\Omega}{=} \bar{x}$ for any point $\bar{x} \in[x]$.

Now, we assume that there is another near fixed point $\bar{x}$ of $T$ satisfying $\bar{x} \notin[x]$, i.e., $\bar{x} \stackrel{\Omega}{=} T(\bar{x})$. Then

$$
\bar{x} \oplus \omega_{1}=T(\bar{x}) \oplus \omega_{2} \text { and } x \oplus \omega_{3}=T(x) \oplus \omega_{4}
$$

for some $\omega_{i} \in \Omega, i=1, \cdots, 4$. Since $T$ is a contraction of $X$ and $d$ satisfies the null equality, we obtain

$$
d(\bar{x}, x)=d\left(\bar{x} \oplus \omega_{1}, x \oplus \omega_{3}\right)=d\left(T(\bar{x}) \oplus \omega_{2}, T(x) \oplus \omega_{4}\right)=d(T(\bar{x}), T(x)) \leq \alpha d(\bar{x}, x),
$$

which implies $d(\bar{x}, x)=0$, since $0<\alpha<1$. Therefore, we obtain $\bar{x} \stackrel{\Omega}{=} x$, which contradicts $\bar{x} \notin[x]$. This says that any $\bar{x} \notin[x]$ cannot be a near fixed point. Equivalently, if $\bar{x}$ is a near fixed point of $T$, then $\bar{x} \in[x]$. This completes the proof. 
Example 8. Continued from Example 5, we see that $(\mathcal{I}, d)$ is a complete informal metric space, such that $d$ satisfies the null equality. Suppose that $T$ is a contraction of $\mathcal{I}$. Then, there exists a real number $0<\alpha<1$ such that

$$
\left.d\left(T\left(\left[a_{1}^{L}, a_{1}^{U}\right]\right), T\left(a_{2}^{L}, a_{2}^{U}\right]\right)\right) \leq \alpha \cdot d\left(\left[a_{1}^{L}, a_{1}^{U}\right],\left[a_{2}^{L}, a_{2}^{U}\right]\right)
$$

for any $\left[a_{1}^{L}, a_{1}^{U}\right],\left[a_{2}^{L}, a_{2}^{U}\right] \in \mathcal{I}$. Given any initial element $I_{0}=\left[a_{0}^{L}, a_{0}^{U}\right] \in \mathcal{I}$, we can generate the iterative sequence $\left\{I_{n}\right\}_{n=1}^{\infty}$ using the function $T$, where $I_{n}=\left[a_{n}^{L}, a_{n}^{U}\right]$, as follows:

$$
I_{1}=T\left(I_{0}\right), \quad I_{2}=T\left(I_{1}\right)=T^{2}\left(I_{0}\right), \cdots, I_{n}=T^{n}\left(I_{0}\right) ;
$$

that is,

$$
\left[a_{1}^{L}, a_{1}^{U}\right]=T\left(\left[a_{0}^{L}, a_{0}^{U}\right]\right), \quad\left[a_{2}^{L}, a_{2}^{U}\right]=T\left(\left[a_{1}^{L}, a_{1}^{U}\right]\right)=T^{2}\left(\left[a_{0}^{L}, a_{0}^{U}\right]\right), \cdots,\left[a_{n}^{L}, a_{n}^{U}\right]=T^{n}\left(\left[a_{0}^{L}, a_{0}^{U}\right]\right) .
$$

Using Theorem 1, the near fixed point $I=\left[a^{L}, a^{U}\right]$ is obtained by the limit

$$
d\left(I_{n}, I\right)=d\left(\left[a_{n}^{L}, a_{n}^{U}\right],\left[a^{L}, a^{U}\right]\right) \rightarrow 0 \text { as } n \rightarrow \infty .
$$

Definition 10. Let $(X, d)$ be an informal metric space with the null set $\Omega$, and let $\Omega$ be closed under the vector addition. A function $T:(X, d) \rightarrow(X, d)$ is called a weakly strict contraction of $X$ if and only if the following conditions are satisfied:

- $\quad x \stackrel{\Omega}{=} y$, i.e., $[x]=[y]$ implies $d(T(x), T(y))=0$;

- $\quad x \neq y$, i.e., $[x] \neq[y]$ implies $d(T(x), T(y))<d(x, y)$.

We see that if $T$ is a contraction of $X$, then it is also a weakly strict contraction of $X$.

Theorem 2. Let $(X, d)$ be an informal, complete metric space with the null set $\Omega$, and let $\Omega$ be closed under the vector addition. Suppose that the function $T:(X, d) \rightarrow(X, d)$ is a weakly strict contraction of $X$. If $\left\{T^{n}\left(x_{0}\right)\right\}_{n=1}^{\infty}$ forms a Cauchy sequence for some $x_{0} \in X$, then $T$ has a near fixed point $x \in X$ satisfying $T(x) \stackrel{\Omega}{=} x$. More precisely, the near fixed point $x$ can be obtained by the following limit

$$
d\left(T^{n}\left(x_{0}\right), x\right) \rightarrow 0 \text { as } n \rightarrow \infty
$$

Assume further that d satisfies the null equality. Then, we also have the following properties.

- There is a unique equivalence class $[x]$ satisfying that if $\bar{x} \notin[x]$ then $\bar{x}$ cannot be a near fixed point, which shows the sense of uniqueness.

- Suppose that $\bar{x} \in[x]$. Then $\bar{x}$ is also a near fixed point of $T$, satisfying $T(\bar{x}) \stackrel{\Omega}{=} \bar{x}$ and $[\bar{x}]=[x]$.

- Suppose that $\bar{x}$ is a near fixed point of T. Then $\bar{x} \in[x]$; i.e., $[\bar{x}]=[x]$. In other words, if $x$ and $\bar{x}$ are the near fixed points of $T$, then $x \stackrel{\Omega}{=} \bar{x}$.

Proof. The assumption says that $\left\{T^{n}\left(x_{0}\right)\right\}_{n=1}^{\infty}$ is a Cauchy sequence. Since $X$ is complete, it follows that $x \in X$ exists, such that $d\left(T^{n}\left(x_{0}\right), x\right) \rightarrow 0$. From Definition 6 and Proposition 2, we see that $T^{n}\left(x_{0}\right) \rightarrow[x]$. Now, given any $\epsilon>0$, there exists an integer $N$, such that $d\left(T^{n}\left(x_{0}\right), x\right)<\epsilon$ for $n \geq N$. Two cases will be considered.

- $\quad$ Suppose that $T^{n}\left(x_{0}\right) \stackrel{\Omega}{=} x$. Since $T$ is a weakly strict contraction of $X$, it follows that

$$
d\left(T^{n+1}\left(x_{0}\right), T(x)\right)=0<\epsilon .
$$

- Suppose that $T^{n}\left(x_{0}\right) \stackrel{\Omega}{\neq} x$. Since $T$ is a weakly strict contraction of $X$, we have

$$
d\left(T^{n+1}\left(x_{0}\right), T(x)\right)<d\left(T^{n}\left(x_{0}\right), x\right)<\epsilon \text { for } n \geq N
$$


Therefore, we conclude that $d\left(T^{n+1}\left(x_{0}\right), T(x)\right) \rightarrow 0$. The triangle inequality says that

$$
d(T(x), x) \leq d\left(T(x), T^{n+1}\left(x_{0}\right)\right)+d\left(T^{n+1}\left(x_{0}\right), x\right) \rightarrow 0 \text { as } n \rightarrow \infty .
$$

Therefore, we obtain $d(T(x), x)=0$, i.e., $T(x) \stackrel{\Omega}{=} x$. This shows that $x$ is a near fixed point.

Now, we assume further that $d$ satisfies the null equality. We want to show that each point $\bar{x} \in[x]$ is a near fixed point of $T$. Since $\bar{x} \stackrel{\Omega}{=} x$, we have $\bar{x} \oplus \omega_{1}=x \oplus \omega_{2}$ for some $\omega_{1}, \omega_{2} \in \Omega$. The null equality says that

$$
d\left(T^{n}\left(x_{0}\right), \bar{x}\right)=d\left(T^{n}\left(x_{0}\right), \bar{x} \oplus \omega_{1}\right)=d\left(T^{n}\left(x_{0}\right), x \oplus \omega_{2}\right)=d\left(T^{n}\left(x_{0}\right), x\right) \rightarrow 0 \text { as } n \rightarrow \infty .
$$

Therefore, we can also obtain $d\left(T^{n+1}\left(x_{0}\right), T(\bar{x})\right) \rightarrow 0$ as $n \rightarrow \infty$ by using the above argument. On the other hand, the triangle inequality also says that

$$
d(\bar{x}, T(\bar{x})) \leq d\left(\bar{x}, T^{n+1}\left(x_{0}\right)\right)+d\left(T^{n+1}\left(x_{0}\right), T(\bar{x})\right) \rightarrow 0 \text { as } n \rightarrow \infty,
$$

which implies $d(\bar{x}, T(\bar{x}))=0$. Therefore, we obtain $T(\bar{x}) \stackrel{\Omega}{=} \bar{x}$ for any point $\bar{x} \in[x]$.

Suppose that $\bar{x} \notin[x]$ and $\bar{x}$ is another near fixed point of $T$. Then, we have $T(\bar{x}) \stackrel{\Omega}{=} \bar{x}$ and $[\bar{x}] \neq[x]$, i.e., $x \nRightarrow \bar{x}$. We also have $T(x) \oplus \omega_{1}=x \oplus \omega_{2}$ and $T(\bar{x}) \oplus \omega_{3}=\bar{x} \oplus \omega_{4}$, where $\omega_{i} \in \Omega$ for $i=1,2,3,4$. Now, we obtain

$$
\begin{aligned}
d(x, \bar{x}) & =d\left(x \oplus \omega_{2}, \bar{x} \oplus \omega_{4}\right) \text { (using the concept of null equality) } \\
& =d\left(T(x) \oplus \omega_{1}, T(\bar{x}) \oplus \omega_{3}\right)=d(T(x), T(\bar{x})) \text { (using the concept of null equality) } \\
& <d(x, \bar{x}) \text { (since } T \text { is a weakly strict contraction and } x \nRightarrow \bar{x} \text { ). }
\end{aligned}
$$

Therefore we led to a contradiction, which says that $\bar{x}$ cannot be a near fixed point of $T$. In other words, if $\bar{x}$ is a near fixed point of $T$, then $\bar{x} \in[x]$. This completes the proof.

Meir and Keeler [11] studied the fixed point theorem for the weakly-uniformly strict contraction. Therefore, under the informal metric space $(X, d)$, we propose the following definition by considering the fact $d(x, y)=0$ for $x \stackrel{\Omega}{=} y$.

Definition 11. Let $(X, d)$ be an informal metric space with the null set $\Omega$, and let $\Omega$ be closed under the vector addition. A function $T:(X, d) \rightarrow(X, d)$ is called a weakly uniformly strict contraction of $X$ if and only if the following conditions are satisfied:

- $\quad x \stackrel{\Omega}{=} y$, i.e., $[x]=[y]$ implies $d(T(x), T(y))=0$;

- given any $\epsilon>0, \delta>0$ exists, such that $\epsilon \leq d(x, y)<\epsilon+\delta$ implies $d(T(x), T(y))<\epsilon$ for any $x \neq y$, i.e., $[x] \neq[y]$.

Remark 3. It is clear to see that if $T$ is a weakly uniformly strict contraction of $X$, then it is also a weakly strict contraction of $X$.

Lemma 1. Let $(X, d)$ be an informal metric space with the null set $\Omega$, and let $\Omega$ be closed under the vector addition. Let $T:(X, d) \rightarrow(X, d)$ be a weakly uniformly strict contraction of $X$. Then the sequence $\left\{d\left(T^{n}(x), T^{n+1}(x)\right)\right\}_{n=1}^{\infty}$ is decreasing to zero for any $x \in X$.

Proof. For convenience, we write $T^{n}(x)=x_{n}$ for all $n$. Let $c_{n}=d\left(x_{n}, x_{n+1}\right)$.

- Suppose that $\left[x_{n-1}\right] \neq\left[x_{n}\right]$. By Remark 3, we have

$$
c_{n}=d\left(x_{n}, x_{n+1}\right)=d\left(T^{n}(x), T^{n+1}(x)\right)<d\left(T^{n-1}(x), T^{n}(x)\right)=d\left(x_{n-1}, x_{n}\right)=c_{n-1} .
$$


- Suppose that $\left[x_{n-1}\right]=\left[x_{n}\right]$. Then, by the first condition of Definition 11,

$$
c_{n}=d\left(T^{n}(x), T^{n+1}(x)\right)=d\left(T\left(x_{n-1}\right), T\left(x_{n}\right)\right)=0<c_{n-1} .
$$

Therefore, we conclude that the sequence $\left\{c_{n}\right\}_{n=1}^{\infty}$ is decreasing. Now, we also consider the following two cases.

- Let $m$ be the first index in the sequence $\left\{x_{n}\right\}_{n=1}^{\infty}$ such that $\left[x_{m-1}\right]=\left[x_{m}\right]$. Then, we can show that $c_{m-1}=c_{m}=c_{m+1}=\cdots=0$. Since $x_{m-1} \stackrel{\Omega}{=} x_{m}$, we have $c_{m-1}=d\left(x_{m-1}, x_{m}\right)=0$. The first condition of Definition 11 says that

$$
0=d\left(T\left(x_{m-1}\right), T\left(x_{m}\right)\right)=d\left(T^{m}(x), T^{m+1}(x)\right)=d\left(x_{m}, x_{m+1}\right)=c_{m},
$$

which implies $x_{m} \stackrel{\Omega}{=} x_{m+1}$; i.e., $\left[x_{m}\right]=\left[x_{m+1}\right]$. We can similarly obtain $c_{m+1}=0$ and $\left[x_{m+1}\right]=$ $\left[x_{m+2}\right]$. Therefore, the sequence $\left\{c_{n}\right\}_{n=1}^{\infty}$ is decreasing to zero.

- Suppose that $\left[x_{m+1}\right] \neq\left[x_{m}\right]$ for all $m \geq 1$. Since the sequence $\left\{c_{n}\right\}_{n=1}^{\infty}$ is decreasing, we can assume that $c_{n} \downarrow \epsilon>0$, i.e., $c_{n} \geq \epsilon>0$ for all $n$, which says that $\delta>0$ exists, such that $\epsilon \leq c_{m}<\epsilon+\delta$ for some $m$, i.e., $\epsilon \leq d\left(x_{m}, x_{m+1}\right)<\epsilon+\delta$. The second condition of Definition 11 says that

$$
c_{m+1}=d\left(x_{m+1}, x_{m+2}\right)=d\left(T^{m+1}(x), T^{m+2}(x)\right)=d\left(T\left(x_{m}\right), T\left(x_{m+1}\right)\right)<\epsilon,
$$

which contradicts $c_{m+1} \geq \epsilon$.

This completes the proof.

Theorem 3. Let $(X, d)$ be an informal complete metric space with the null set $\Omega$, and let $\Omega$ be closed under the vector addition. Let $T:(X, d) \rightarrow(X, d)$ be a weakly uniformly strict contraction of $X$. Then $T$ has a near fixed point satisfying $T(x) \stackrel{\Omega}{=} x$. More precisely, the near fixed point $x$ is obtained by the following limit

$$
d\left(T^{n}\left(x_{0}\right), x\right) \rightarrow 0 \text { as } n \rightarrow \infty \text { for some } x_{0} .
$$

Assume further that d satisfies the null equality. Then we also have the following properties.

- There is a unique equivalence class $[x]$ satisfying that if $\bar{x} \notin[x]$, then $\bar{x}$ cannot be a near fixed point, which shows the sense of uniqueness.

- Suppose that $\bar{x} \in[x]$. Then $\bar{x}$ is also a near fixed point of $T$ satisfying $T(\bar{x}) \stackrel{\Omega}{=} \bar{x}$ and $[\bar{x}]=[x]$.

- Suppose that $\bar{x}$ is a near fixed point of T. Then $\bar{x} \in[x]$; i.e., $[\bar{x}]=[x]$. In other words, if $x$ and $\bar{x}$ are the near fixed points of $T$, then $x \stackrel{\Omega}{=} \bar{x}$.

Proof. From Theorem 2 and Remark 3, we just need to show that if $T$ is a weakly uniformly strict contraction, then $\left\{T^{n}\left(x_{0}\right)\right\}_{n=1}^{\infty}=\left\{x_{n}\right\}_{n=1}^{\infty}$ is a Cauchy sequence for $x_{0} \in X$. Suppose that $\left\{x_{n}\right\}_{n=1}^{\infty}$ is not a Cauchy sequence. By definition, $2 \epsilon>0$ exists, such that, given any $N, m, n \geq N$ exists, satisfying $d\left(x_{m}, x_{n}\right)>2 \epsilon$. The assumption says that $T$ is a weakly uniformly strict contraction on $X$. Therefore, $\delta>0$ exists, such that

$$
\epsilon \leq d(x, y)<\epsilon+\delta \text { implies } d(T(x), T(y))<\epsilon \text { for any } x \not y .
$$

Let $\delta^{\prime}=\min \{\delta, \epsilon\}$. We want to show that

$$
\epsilon \leq d(x, y)<\epsilon+\delta^{\prime} \text { implies } d(T(x), T(y))<\epsilon \text { for any } x \nRightarrow y .
$$

It is clear to see that if $\delta^{\prime}=\epsilon$, i.e., $\epsilon<\delta$, then $\epsilon+\delta^{\prime}=\epsilon+\epsilon<\epsilon+\delta$. 
Let $c_{n}=d\left(x_{n}, x_{n+1}\right)$. Lemma 1 says that the sequence $\left\{c_{n}\right\}_{n=1}^{\infty}$ is decreasing to zero. Therefore, we can find $N$ such that $c_{N}<\delta^{\prime} / 3$. For $n>m \geq N$, we have

$$
d\left(x_{m}, x_{n}\right)>2 \epsilon \geq \epsilon+\delta^{\prime},
$$

which implicitly says that $x_{m} \supsetneqq x_{n}$. Since $\left\{c_{n}\right\}_{n=1}^{\infty}$ is decreasing, we obtain

$$
d\left(x_{m}, x_{m+1}\right)=c_{m} \leq c_{N}<\frac{\delta^{\prime}}{3} \leq \frac{\epsilon}{3}<\epsilon .
$$

For $j$ with $m<j \leq n$, we also have

$$
d\left(x_{m}, x_{j+1}\right) \leq d\left(x_{m}, x_{j}\right)+d\left(x_{j}, x_{j+1}\right) .
$$

We want to show that $j$ with $m<j \leq n$ exists, such that $x_{m} \nRightarrow x_{j}$ and

$$
\epsilon+\frac{2 \delta^{\prime}}{3}<d\left(x_{m}, x_{j}\right)<\epsilon+\delta^{\prime}
$$

Let $\gamma_{j}=d\left(x_{m}, x_{j}\right)$ for $j=m+1, \cdots, n$. Then (6) and (7) say that

$$
\gamma_{m+1}<\epsilon \text { and } \gamma_{n}>\epsilon+\delta^{\prime} .
$$

Let $j_{0}$ be an index satisfying

$$
j_{0}=\max \left\{j \in[m+1, n]: \gamma_{j} \leq \epsilon+\frac{2 \delta^{\prime}}{3}\right\} .
$$

Using (10), we have $m+1 \leq j_{0}<n$. This says that $j_{0}$ is well-defined. The definition of $j_{0}$ also says that $j_{0}+1 \leq n$ and $\gamma_{j_{0}+1}>\epsilon+\frac{2 \delta^{\prime}}{3}$. Therefore, we obtain $x_{m} \nRightarrow x_{j_{0}+1}$, which says that the expression (9) will be sound if we can show that

$$
\epsilon+\frac{2 \delta^{\prime}}{3}<\gamma_{j_{0}+1}<\epsilon+\delta^{\prime}
$$

Suppose that this is not true; i.e., $\gamma_{j_{0}+1} \geq \epsilon+\delta^{\prime}$. Using (8), we obtain

$$
\frac{\delta^{\prime}}{3}>c_{N} \geq c_{j_{0}}=d\left(x_{j_{0}}, x_{j_{0}+1}\right) \geq \gamma_{j_{0}+1}-\gamma_{j_{0}} \geq \epsilon+\delta^{\prime}-\epsilon-\frac{2 \delta^{\prime}}{3}=\frac{\delta^{\prime}}{3}
$$

which contradicts the fact that (9) is sound. Since $x_{m} \neq x_{j}$, forms (5), we see that (9) implies

$$
d\left(x_{m+1}, x_{j+1}\right)=d\left(T\left(x_{m}\right), T\left(x_{j}\right)\right)<\epsilon .
$$

Therefore, we obtain

$$
\begin{aligned}
d\left(x_{m}, x_{j}\right) & \leq d\left(x_{m}, x_{m+1}\right)+d\left(x_{m+1}, x_{j+1}\right)+d\left(x_{j+1}, x_{j}\right) \\
& <c_{m}+\epsilon+c_{j}(\text { by }(11)) \\
& <\frac{\delta^{\prime}}{3}+\epsilon+\frac{\delta^{\prime}}{3}=\epsilon+\frac{2 \delta^{\prime}}{3}
\end{aligned}
$$

which contradicts (9). Therefore, every sequence $\left\{T^{n}(x)\right\}_{n=1}^{\infty}=\left\{x_{n}\right\}_{n=1}^{\infty}$ is a Cauchy sequence. This completes the proof. 
Funding: This research received no external funding.

Conflicts of Interest: The author declares no conflict of interest.

\section{References}

1. Adasch, N.; Ernst, B.; Keim, D. Topological Vector Spaces: The Theory without Convexity Conditions; Springer-Verlag: Berlin/Heidelberg, Germany, 1978.

2. Khaleelulla, S.M. Counterexamples in Topological Vector Spaces; Springer-Verlag: Berlin/Heidelberg, Germany, 1982.

3. Schaefer, H.H. Topological Vector Spaces; Springer-Verlag: Berlin/Heidelberg, Germany, 1966.

4. Peressini, A.L. Ordered Topological Vector Spaces; Harper and Row: New York, NY, USA, 1967.

5. Wong, Y.-C.; Ng, K.-F. Partially Ordered Topological Vector Spaces; Oxford University Press: Oxford, UK, 1973.

6. Wu, H.-C. Near Fixed Point Theorems in Hyperspaces. Mathematics 2018, 6, 90. [CrossRef]

7. Wu, H.-C. Near Fixed Point Theorems in the Space of Fuzzy Numbers. Mathematics 2018, 6, 108. [CrossRef]

8. Aubin, J.-P. Applied Functional Analysis, 2nd ed.; John Wiley \& Sons: Hoboken, NJ, USA, 2000.

9. Conway, J.B. A Course in Functional Analysis, 2nd ed.; Springer-Verlag: Berlin/Heidelberg, Germany, 1990.

10. Riesz, F.; Sz.-Nagy, B. Functional Analysis; Dover Publications, Inc.: New York, NY, USA, 1955.

11. Meir, A.; Keeler, E. A Theorem on Contraction Mappings. J. Math. Anal. Appl. 1969, 28, 326-329. [CrossRef]

(C) 2019 by the authors. Licensee MDPI, Basel, Switzerland. This article is an open access article distributed under the terms and conditions of the Creative Commons Attribution (CC BY) license (http:/ / creativecommons.org/licenses/by/4.0/). 